\section{R. Heij}

\section{A. G. Steel}

P.J.Young

Queen Elizabeth Hospital NHS Foundation Trust, King's Lynn, UK

E-mail: alistair.steel@nhs.net

No competing interests declared.
2. Cook TM. Personal protective equipment during the COVID-19 pandemic - a narrative review. Anaesthesia 2020; 75: 920-7.

3. Wang D, Hu B, Hu C, et al. Clinical characteristics of 138 hospitalized patients with 2019 novel coronavirus-infected pneumonia in Wuhan, China. Journal of the American Medical Association 2020; 323: 1061-9.

4. Kwok YLA, Gralton J, McLaws ML. Face touching: a frequent habit that has implications for hand hygiene. American Journal of Infection Control 2015; 43: 112-4.

\title{
References
}

1. Public Health England. Recommended PPE for healthcare workers by secondary care inpatient clinical setting, NHS and independent sector. v1.02-04-2020 (accessed 04/04/20).

\section{The Utah model: mental bandwidth and strategic risk generation in COVID-19 airway management}

We would like to thank Cook et al. for their consensus guidelines for managing the airway in patients with COVID19 [1].

Airway management in the COVID-19 patient is a point of risk concentration for providers, patients and medical systems. Time is of the essence if medical systems are to turn the tide of collapse seen in Wuhan, Italy, Spain and now, New York. We would like to highlight two points arising from our reading of these guidelines. First, failure to minimise aggregate airway management risk poses a strategic threat to our medical systems; and two, it is critical to include the risk of mental bandwidth saturation as a risk for contamination. In addition, we offer a simple, qualitative, mental model that explains how the risk of provider exposure is generated during airway management.

Complex medical systems are required to fight complex diseases. Where medical systems are intact, COVID-19 mortality is 1-2\%; where collapsed, mortality is 6-10\% [2]. We are moving rapidly towards a resource-constrained medical system. In a resource-constrained system, providers are the most critical resource. Providers enable the effectiveness of tactics and tools deployed to fight disease. Delivering complex care is not possible without providers. The first principle of the resource-constrained system is 'protect your providers: they are the system'.

When the aggregate disease burden exceeds aggregate medical system resources, mortality increases. Airway management has an outsized impact on burden and resources. For example, multiple intubation attempts correlate with an increase in cardiac arrest, hypoxaemia, hypotension and aspiration, as well as ICU mortality [3].
Failure to minimise aggregate airway management risk contributes to a resource-constrained medical system. It is critical to include the risk of mental bandwidth saturation into our basic provider exposure risk generation model. The link between increasing mental task load and performance is well-described [4]. Initially, as stress increases, performance increases until mental bandwidth is saturated, after which performance deteriorates.

Tracheal intubation, especially when difficult, is a high mental task load procedure. In a critically ill patient, with personal protection equipment (PPE) shortage, and the general stress of a pandemic, one can see how quickly mental bandwidth saturation occurs. We believe that increasing mental task-load can lead to an increased likelihood of non-adherence to infectious disease protocols, increasing the risk of provider exposure.

The Utah model is designed to facilitate a common understanding of the existential risks to providers, the medical system and the populations arising from airway management in COVID-19 patients. In our qualitative model, the vertical axis indicates increasing aerosolisation risk; the horizontal axis is increasing mental task-load. The grid formed can then be interpolated as semicircular zones of aggregate elevated provider exposure risk (Fig. 1). As examples, we have placed various airway procedures on the Utah model. The relationships are purely the expert opinion of the authors. Others might place them differently. Note that we can now discuss those differences as we are working from a common mental model of risk generation.

We acknowledge that the Utah model is imperfect, low fidelity and depends on a foundation of expert opinion and 


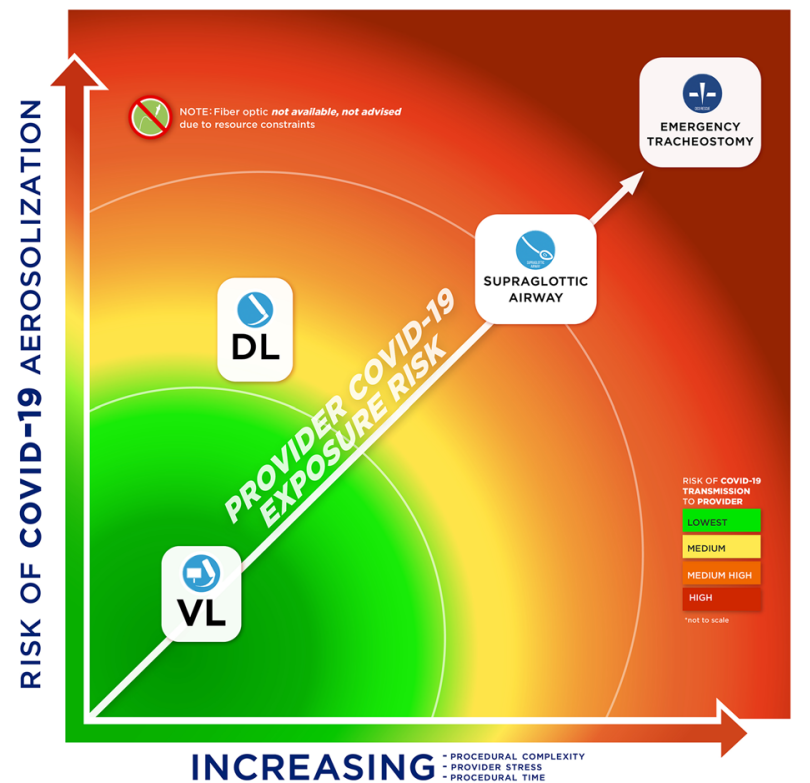

Figure 1 A provider exposure risk model.

anecdotal observation, with only a little scientific evidence. We believe this model can catalyse shared understanding, speed consensus, and perhaps adherence to airway protocols in order to optimise aggregate risk.

Time is of the essence; every attempt, complication and infected provider, counts in the fight against COVID-19.

\author{
S. Runnels \\ D. Ferranti \\ A.N. Davis \\ J. Pollard \\ University of Utah, \\ Salt Lake City, UT, USA \\ Email: runnelss@yahoo.com
}

No competing interests declared.

\section{References}

1. Cook TM, El-Boghdadly K, McGuire B, McNarry AF, Patel A, Higgs $A$. Consensus guidelines for managing the airway in patients with COVID-19. Anaesthesia 2020; 75: 785-99.

2. Coronavirus Cases. Worldometer, 2020. https://www.world ometers.info/coronavirus/coronavirus-cases/\#daily-cases (acces sed 29/03/2020).

3. Spaite DW, Hu C, Bobrow BJ, et al. Association of out-of-hospital hypotension depth and duration with traumatic brain injury mortality. Annals of Emergency Medicine 2017; 70: 522-30.e1.

4. Bourne Jr LE, Yaroush RA. Stress and cognition: a cognitive psychological perspective. NASA Technical Reports Server 2003: 1-123.

doi:10.1111/anae.15086 